ÉGYPTE monde arabe

\section{Égypte/Monde arabe}

12-13 | 1993

Une économie en transition

\title{
Les perceptions de la violence politique en Égypte. Présentation
}

\section{François Burgat et Baudouin Dupret}

\section{(2) OpenEdition}

1 Journals

Édition électronique

URL : https://journals.openedition.org/ema/1272

DOI : 10.4000/ema. 1272

ISSN : 2090-7273

\section{Éditeur}

CEDEJ - Centre d'études et de documentation économiques juridiques et sociales

\section{Édition imprimée}

Date de publication : 31 mars 1993

Pagination : 243-250

ISSN : 1110-5097

\section{Référence électronique}

François Burgat et Baudouin Dupret, « Les perceptions de la violence politique en Égypte.

Présentation », Égypte/Monde arabe [En ligne], 12-13 | 1993, mis en ligne le 08 juillet 2008, consulté le 07 juillet 2022. URL : http://journals.openedition.org/ema/1272 ; DOl : https://doi.org/10.4000/ema. 1272

Ce document a été généré automatiquement le 7 juillet 2022.

Tous droits réservés 


\title{
Les perceptions de la violence politique en Égypte. Présentation
}

\author{
François Burgat et Baudouin Dupret
}

1 L'assassinat de Farag Fawda l'a relancée ; les récurrentes émeutes de Haute-Égypte puis du Caire lui ont donné un nouveau souffle; le recours, à partir du mois de juillet, aux attentats aveugles (notamment contre les touristes) et le surcroît de répression qu'ils ont engendré en ont - si cela était encore possible - fait une nouvelle fois monter le ton : l'Égypte politique et intellectuelle vit à bien des égards depuis plus d'une année mais en fait bien davantage - à l'heure du débat sur la violence politique ${ }^{1}$ et le terrorisme.

\section{Les représentations de la violence}

Les lignes qui suivent n'ont pas l'ambition d'aborder de manière construite et exhaustive cette question centrale du débat politique en cours. Plus modestement, elles se proposent de donner à lire quelques échantillons des problématiques abordées dans la presse par les observateurs et/ou les protagonistes de ce débat, tout en réintroduisant certaines perspectives politiques partiellement absentes du débat officiel local ou de ses échos dans la presse étrangère. Quantitativement, le matériau disponible était surabondant sinon franchement pléthorique. Dans une série intitulée «Le terrorisme et l'extrémisme dans la pensée des intellectuels » et à l'exclusion de ses abondants éditoriaux, le seul quotidien Al-Ahrâm avait publié, au début de l'année 1993, plus d'une centaine d'analyses ou prises de positions en tous genres. Tous les organes de presse - officiels, officieux ou d'opposition - en ont fait autant et le thème du terrorisme est sans doute celui qui leur a fourni, à ce jour, le plus de manchettes et autres couvertures.

3 En janvier 1993, la traditionnelle Foire internationale du livre a mobilisé le public lors de conférences quotidiennes pour entendre les ténors de la scène intellectuelle et médiatique dénoncer (à l'instar des larges placards publicitaires bilingues apparus un peu partout dans la ville) le «terrorisme islamique $»^{2}$. Hommes ou femmes, « laïcs » ou 
" religieux », hommes de pouvoir ou opposants, chrétiens ou musulmans, militaires ou civils, artistes ou hommes de lois, universitaires de toutes disciplines, il est en apparence peu de catégories théoriques de la société égyptienne qui n'aient été, à un degré ou à un autre, sollicitées par l'appareil médiatique.

Pour rendre compte des éléments de ce débat, des choix forcément subjectifs ont donc été opérés. D'abord pour des raisons de lisibilité et d'intérêt. Ensuite, du fait d'une certaine réticence des acteurs du débat « officiel » à prendre en compte la dimension strictement politique du problème de la violence, et notamment les piètres performances du système institutionnel en matière de représentation. Bon nombre de démarches explicatives ont eu en effet tendance à se cantonner aux seules expressions confessionnelles de cette violence. Cette "surconfessionalisation " aboutissait ainsi à occulter la fonctionnalité réelle d'une large majorité des occurrences de violence (qui n'opposent pas l'une des communautés religieuses à l'autre mais le régime à une partie de son opposition politique) en même temps qu'elle reléguait au second plan une perspective explicative (l'effondrement de la participation politique) qui est à l'évidence autrement plus centrale que les différends confessionnels, aussi réels soientils. L'immense majorité des quelque cent intervenants de la première série d'interventions publiées par Al-Ahrâm ${ }^{3}$ s'est ainsi tenue significativement à l'écart de la critique de cette dichotomie croissante entre la réalité des forces politiques en présence et leur représentation par l'institution parlementaire. L'importance de la répression et sa fonctionnalité dans l'enchaînement des conduites violentes n'ont, par ailleurs, pas toujours trouvé leur juste place dans l'inventaire des causalités.

5 La presse d'opposition, eu égard à la coloration « islamique » des protagonistes (autres qu'étatiques) de la violence, s'est elle-même scindée en deux camps assez tranchés. Les voix d'une gauche élue lors d'élections boycottées par les (ou interdites aux) islamistes, et qui sait qu'elle a peut-être tout à perdre et certainement rien à gagner à une transparence accrue du système parlementaire, n'ont pas renvoyé d'écho très différent $\mathrm{du}$ discours officiel. Le thème du terrorisme a fourni au contraire au régime l'occasion d'un spectaculaire rapprochement avec les partis de l'opposition laïque (Wafd et Tagammu'). Le magazine Rose al-Youssef, porte-parole de la gauche ex-nassérienne, l'hebdomadaire al-Ahâli (organe du Tagammu' de Khaled Mohieddine) et le quotidien alWafd ont œuvré sur un registre à peu près identique à celui des organes de la presse officielle tels qu'Al-Ahrâm, October, Al-Musawwar, al-Akhbar, Akhir Sâ'a etc., cherchant notamment à impliquer la composante légaliste du courant islamiste dans les violences contre les touristes. Quant aux journaux à tendance islamiste Al-Cha'b, Al-Nûr, Al-Haqîqa, Al-Mukhtar al-lslâmi, ils ont tenté pour leur part, tout en condamnant le recours à la violence contre les touristes, de circonscrire la responsabilité des attentats à la frange radicale du courant et à réintroduire plus systématiquement dans l'analyse la répression policière et les limites du système institutionnel.

6 À défaut de désigner les faiblesses du dispositif électoral de légitimation de l'État égyptien, l'opposition laïque a mis en cause ses politiques sectorielles - le système éducatif et certaines des valeurs qu'il véhicule, les moyens d'information audiovisuels notamment (et la place qu'ils donnent aux tenants d'une vision populiste et démagogique de l'Islam) ou encore - et surtout - une politique économique et sociale dont l'échec est considéré par l'immense majorité des commentateurs comme le paramètre central de toute analyse des conduites violentes. Refusant eux aussi la lecture politique des enjeux en cause, les représentants de l'Islam institutionnel ont le 
plus souvent consacré leurs efforts à souligner la pureté originelle d'un Islam désincarné et intemporel vis-à-vis de toute forme de violence, confessionnelle en particulier ou politique en général.

7 La presse dite islamiste a largement participé au débat. Elle n'est toutefois représentative de cette vaste mouvance que de manière assez inégale, l'accès aux médias, malgré un climat de relative liberté ${ }^{4}$ - demeurant en fait assez sélectif. La sensibilité du Parti du Travail dispose, avec le bihebdomadaire Al-Cha'b, d'un puissant instrument de communication que la crise du Golfe a hissé, par son tirage, au premier rang de la presse d'opposition ${ }^{5}$. Les Frères Musulmans, même si leur présence est acquise à titre individuel et officieux dans divers médias dont $A l-C h a^{\prime} b^{6}$ ne disposent par contre, en l'absence de statut légal, d'aucun titre où ils puissent s'exprimer en tant que parti.

8 La composante la moins présente sur le marché de l'écrit se trouve paradoxalement être celle qui est impliquée le plus directement dans la spirale de la violence. Les Gamâ'ât Islâmiyya - dont l'immense majorité des leaders sont emprisonnés et le reste dans la clandestinité ou l'exil - ne s'expriment qu'exceptionnellement par voie de presse $^{7}$. Hormis quelques extraits d'entretiens téléphoniques avec l'un de leurs leaders symboliques, 'Umar 'Abd al-Rahman, les «terroristes » eux-mêmes ou leur famille politique directe n'ont donc que rarement exprimé un point de vue, la plus importante exception étant un long communiqué publié dans le courant du mois de décembre par $a l-C h a^{\prime} b^{8}$. Dans plusieurs cas il est vrai, et notamment à Imbâba, des prévenus présentés comme des leaders du mouvement ont été longuement interrogés à la télévision, mais leur statut réel était, dans ce cas, loin d'être clairement établi. ${ }^{9}$

9 La presse étrangère n'a pas sensiblement renouvelé la problématique officielle mais a eu plutôt tendance, au contraire, à la « radicaliser ». Le 10 octobre, un éditorial de 'Adil Husayn, l'un des leaders du Parti (islamiste) du Travail ayant critiqué, à l'occasion de la réunion au Caire d'une convention d'agents de voyage américains et internationaux (ASTA), le déséquilibre entre les faibles apports économiques du tourisme et l'importance de son impact négatif sur les plans moral et social, les autorités se sont employées à lui faire porter la responsabilité des violences anti-touristes : ainsi a-t-il été entendu en compagnie d'Ibrahim Chukri, leader du Parti du Travail, par la Cour de sûreté de l'état, sous la double accusation d'avoir "poussé à l'émeute " après le tremblement de terre et " incité à la haine contre les touristes » ${ }^{10}$. Cette thèse, reprise par une partie de la presse égyptienne de gauche ou gouvernementale, a été reproduite par certains des organes les plus en vue de l'appareil médiatique étranger. La version qu'en a publié le journal Le Monde donne un bon exemple de cette réappropriation :

«Pour les barbus», écrit le correspondant au Caire du quotidien français, «la paralysie totale du secteur touristique est devenue une stratégie destinée à renverser «le régime impie ». Depuis quelques années, ils ont tout essayé. Ils ont tué de hauts responsables comme le président de l'Assemblée, ils ont égorgé des policiers, mitraillé des penseurs libéraux ou abattu des dizaines de coptes en HauteÉgypte. Cela n'a pas affolé le gouvernement, qui s'est contenté de donner quelques coups de bâton par-ci par-là. Ces attentats n'étaient pas considérés comme "très inquiétants». On en était même arrivé à ne plus vraiment s'en apercevoir. Quel écho peut en effet provoquer l'assassinat d'un policier payé $500 \mathrm{~F}$ par mois, ou d'un copte issu d'une minorité, habitué à courber l'échine en attendant que la tempête passe ? C'est à ce moment qu'un ancien marxiste converti à l'islamisme radical a eu un trait de génie. Il a écrit dans l'hebdomadaire qu'il dirige que le tourisme était harâm (contraire à l'Islam). C'était en été, un été où le tourisme battait tous les 
records avec les hôtels pleins à $80 \%$. La fatwa (avis de jurisprudence islamique) n'est pas tombée dans l'oreille d'un sourd et, pour la première fois de l'histoire de l'Égypte moderne, les touristes sont devenus des cibles. »

10 Même s'il manque dans cette chaîne de causalité plusieurs maillons essentiels ${ }^{11}$, cette grille d'analyse semble s'être imposée au lectorat francophone.

\section{L'économie du politique}

11 L'État est-il, en Égypte, contraint de recourir aux solutions répressives parce qu'il n'existe, dans le paysage politique, aucun acteur capable de jouer - dans le respect des règles du système - le jeu de la participation? Ou bien, à l'inverse, certains éléments du paysage politique se tournent-ils vers la violence - que ce soit pour la pratiquer ou seulement pour la cautionner ou la tolérer - faute de trouver dans le paysage institutionnel la moindre possibilité sérieuse de participation?

La violence anti-chrétienne est-elle un élément structurel de la dynamique de réislamisation dont l'État aurait alors toute légitimité à réprimer la moindre expression politique au nom de la défense de l'« unité nationale »? Ou bien n'est-elle qu'une forme périphérique de la violence contre l'État, qui en amplifie la portée et, ce faisant - au risque de mettre en danger, à terme, la communauté qu'il prétend protéger - tente de cautionner le déploiement répressif qui lui permet de survivre?

On émettra pour notre part l'hypothèse qu'amputée de sa dimension essentielle qu'est l'alternance, l'ouverture politique esquissée en Égypte - notamment par la libéralisation de la presse - tend davantage à exacerber les carences du système de représentation qu'à les résorber. Dans cette hypothèse, le «terrorisme " et les «atteintes à l'unité nationale » nées de la "sédition confessionnelle ", érigés en réponse à toute demande de participation politique, auraient - en partie au moins vocation à exorciser la difficulté croissante du régime à gérer les implications de sa trop grande longévité ${ }^{12}$.

Le danger d'une «surconfessionalisation" des dynamiques politiques a déjà été dénoncé :

«[...] Au cours des années 70 [...], lorsque le gouvernement a voulu se doter d'une législation renforçant ses pouvoirs vis-à-vis de l'opposition, souligne en ce sens l'historien Tariq al-Bichri, vice-président du Conseil d'État ${ }^{13}$, il y est parvenu en prétendant que couvait une discorde confessionnelle ("fitna ta'ifiyya »), et il a promulgué, sous l'appellation de «Loi de l'unité nationale », un texte qui visait en fait toute l'opposition politique. [...]. On sait également que dans les années 80, la lutte contre le courant islamique a cherché à s'attirer le public des adversaires laïques de ce courant en nourrissant la haine entre les coptes et l'Islam politique en général [...]. Les initiateurs d'une telle démarche, qui sont allés jusqu'à demander " moins d'Islam » pour garantir l'« unité » et la sécurité de la société, ont alors joué le même jeu dangereux qu'un marin qui, pour frapper un de ses adversaires, arracherait une planche au fond de son navire. »

L'usage de la rhétorique de l'unité nationale pour légitimer le verrouillage de la représentation politique, s'il ne suffit pas à clore l'inventaire des causalités de la violence, doit donc vraisemblablement y retrouver sa place, et les conséquences de l'incapacité du système institutionnel à intégrer de larges composantes du paysage politique être mesurées avec plus d'attention. C'est ce que fait encore Tariq al-Bichri 
lorsque, évoquant l'existence de ces «forces politiques dépourvues de reconnaissance légale ", il constate que

«chaque fois que dans ses veines on ne laisse plus circuler librement les véritables dynamiques politiques, chaque fois que ses canaux se rétrécissent au point de ne plus assimiler l'ensemble de ces flux, le système fait le lit aux organisations secrètes et autres groupes clandestins [...]. Lorsqu'un système politique en vient à donner le jour à de multiples formations n'ayant aucune relation avec la réalité politique, sociale et culturelle du pays, si ce qui existe légalement n'existe pas réellement alors même que ce qui existe réellement n'a pas la reconnaissance du droit, si un système politique tolère une dichotomie entre le légal et le réel [...], c'est une véritable schizophrénie qui risque d'affecter la société toute entière. »

\section{NOTES}

1. Chaque année, le Rapport stratégique arabe publié par le Centre d'Études stratégiques du journal Al-Ahrâm consacre une rubrique spéciale à « La violence politique en Égypte ». On pourra lire un exemple de cette production (1989) in Égypte/Monde arabe $\mathrm{n}^{\circ} 4$ p. 187.

2. « No to terrorism ".

3. La publication de ce dossier ayant été initialement programmée pour le numéro précédent, le choix de l'essentiel des textes a été opéré en conséquence.

4. Qui n'est pas toutefois sans limite: en Égypte (comme à peu près partout ailleurs dans le monde), c'est la publicité qui assure la santé, financière d'un quotidien et non ses ventes. Dans le cas d'Al-Cha'b, le journal est vendu à perte et sa situation financière est donc inversement proportionnelle à son niveau de diffusion. C'est en faisant pression sur les annonceurs que le régime parvient donc pour une part à limiter la capacité d'expression de l'opposition. L'autre limite, sans doute plus importante, tient au tait que la liberté de la presse est étroitement circonscrite aux supports écrits et - eu égard au taux d'analphabétisme - ne touche donc qu'une faible partie de la population, alors que les grands médias audiovisuels sont entièrement contrôlés par le régime.

5. Le tirage d'Al-Cha'b oscillerait entre 450000 exemplaires (au plus fort de la crise du Golfe) et 200000 au cours de l'année 1992. Depuis le 20 février 1993 paraît une édition internationale éditée aux USA. Deux autres hebdomadaires officiellement rattachés au parti des libéraux et considérés comme proche des saoudiens, Al-Nûr et Al-Haqiqa, évoluent également, quoiqu'avec des lignes éditoriales beaucoup plus fluctuantes, dans la mouvance islamiste semioppositionnelle. Le Centre d'études arabes et islamiques affilié au Parti du Travail publie depuis près de deux ans une revue, Minbar al-Charq, dont l'objectif énoncé est de développer la communication à la fois entre les différentes composantes du courant islamiste, d'une part, et entre ce dernier, les chrétiens et les forces de la gauche dite laïque, d'autre part. Des conférences sont organisées mensuellement (le premier jeudi de chaque mois) au siège de la revue, 18 châri' Ibrâhim Laqâni, à Héliopolis.

6. Cf. par exemple la chronique hebdomadaire de Mustafa Machhûr, un des membres actuellement les plus influents de l'association dans le bi-hebdomadaire al-Cha'b.

7. Le limogeage, en 1990, du ministre de l'intérieur Zaki Badr avait conduit les porte-parole de la Jamâ'a à se risquer à tenir au Caire des conférences de presse et à diffuser plus régulièrement leurs communiqués. La flambée de répression consécutive à l'assassinat du président du 
parlement les a fait plonger à nouveau dans une semi-clandestinité. La revue Al-Murâbitûn, publiée au Pakistan, est considérée comme très proche de la Jamâ'a égyptienne. L'organisation al-Jihâd publie pour sa part (depuis Paris, suppose-t-on) un bulletin sous le titre Al-Fath.

8. Les bribes de discours qui en émanent ont toutefois une certaine unité. Ils s'emploient en général à rejeter sur l'État qui les réprime l'initiative de la violence dans laquelle celui-ci tente de les enfermer. C'est également le registre des réponses que fait le cheikh 'Urnar 'Abd al-Rahman depuis son exil new-yorkais. Cf. notamment deux de ses interviews dans le journal Al-Cha'b: Le 8 juillet 1992, il y déclare que la Jamâ'a islamiyya «n'a jamais eu l'intention de recourir à la violence contre les coptes »et que « seul le gouvernement égyptien profite de la sédition confessionnelle en Égypte». Le 27 novembre, il conditionne la fin de la confrontation avec les autorités égyptiennes à l'ouverture d'un dialogue entre les deux parties et dément une nouvelle fois avoir «donné des instructions pour lutter contre le tourisme ».

9. Ce fut notamment le cas de " cheikh Jaber », que la Jamâ'a ne reconnaît pas comme l'un de ses leaders.

10. «Remous sur le Nil », Le Monde, 28 novembre 1992.

11. La tonalité de l'article de 'Adil Husayn est celle d'une critique économiste assez mesurée du phénomène touristique, et les réticences qu'il émet vis-à-vis de son impact social et moral ont, de longue date, déjà été formulées depuis d'autres horizons politique ou religieux. L'article de l'ancien militant communiste a été ensuite publié non pas " en été », « lorsque le tourisme battait son plein ", mais le 10 octobre, soit plus de deux mois après le début des agressions contre les touristes. Par ailleurs, en réduisant à 'quelques coups de bâton' l'ampleur de la répression croissante contre la Jamâ'a islamiyya, l'analyse se prive d'un point d'ancrage essentiel puisque, pour insuffisantes qu'elles soient, toutes les 'justifications' données par les auteurs des attentats l'ont été en termes de réaction aux traitements (détentions, tortures et parfois éliminations physiques) infligés aux membres ou aux sympathisants des Jamâ'ât et parfois à leurs proches. Ainsi, au cours de l'année 1992, 9428 personnes- fourchette basse des estimations - ont été incarcérées, soit en moyenne 25 chaque jour, et l'usage répété de la torture contre les prévenus et de diverses formes de violence contre leurs familles a été régulièrement dénoncé par différentes instances locales et internationales (Cf. Al-mujtama' al madani wa-l-tahawwul aldîmûkrâti fi-l-watan al-'arabî - La société civile et la transition démocratique dans le monde arabe -, Markaz Ibn Khaldûn, Annual Report 1992, Dâr Sa'd al-Sabah, Le Caire, 1993, 504 p., p. 72, ainsi que le rapport de l'Organisation égyptienne des droits de l'homme: Imbâba, une image intense de la détérioration de l'état des droits de l'homme et du respect de la loi en Égypte (qui recense également les abus attribués aux membres des Jamâ'ât Islamiyya (Le Caire, 20 mars 1993).Quiconque, enfin, a tenté de s'approcher un tant soit peu de la mouvance islamiste égyptienne a pu se convaincre que le statut de 'Adil Husayn, pour important qu'il soit du fait, notamment, de la bonne diffusion du journal qu'il dirige, ne va pas jusqu'à lui conférer un quelconque leadership, ni direct, ni indirect, sur une mouvance activiste des Jamâ'ât qui est bien loin de le reconnaitre. La filiation qui a fait fortune dans la presse étrangère apparaît donc, de ce seul point de vue, comme aussi crédible qu'une interprétation 'arabe' des dynamiques politiques françaises qui aurait associé, dans les années 70, un discours du secrétaire général du Parti communiste français, M. Georges Marchais, à une campagne du groupe extrémiste Action directe, ou une grève de la CGT à une sollicitation de M. Jean Lecanuet.

12. Cf. François Burgat, «Égypte 1990 : les refuges du politique », Annuaire de l'Afrique du Nord 1990, Editions du CNRS, Paris, 1992.

13. Dans son introduction au rapport «L'annuaire de la nation », Égypte/Monde arabe $\mathrm{n}^{\circ} 11$, présentation et traduction F. Burgat et B. Dupret. 
INDEX

Mots-clés : Fawda (Farag), islamisme, terrorisme, violence

\section{AUTEURS}

FRANÇOIS BURGAT

CNRS-Cedej

\section{BAUDOUIN DUPRET}

Cedej 